\title{
Uso del PVC Orientado como herramienta de ahorro energético en las obras de Modernización del Regadío de la Z.R de la C.R del Trasvase Tajo-Segura de Librilla. Sectores 2 y 3
}

\author{
Rincón Benito, Marcos (1) (P)
}

1Asistencia Técnica, Molecor, marcos.rincon@molecor.com

\section{Introducción.}

La modernización del sistema de riego es crucial para el mantenimiento de la actividad agrícola en el ámbito del área regable de la Comunidad de Regantes de Librilla. Su déficit hídrico, consecuencia del estructural que padece el sureste español y del sistema de riego que se emplea en la actualidad, obliga a disponer de uno con la mayor eficiencia posible, que asegure el máximo aprovechamiento de los recursos disponibles. Además, aunque diferentes programas de reabastecimiento de agua estatales o autonómicos fueran capaces de equilibrar el balance hídrico, el coste del $\mathrm{m}^{3}$, como ya está ocurriendo, no permite el mal- gasto del recurso que supone el riego por inundación comparado con otros sistemas de mayor eficiencia intrínseca.

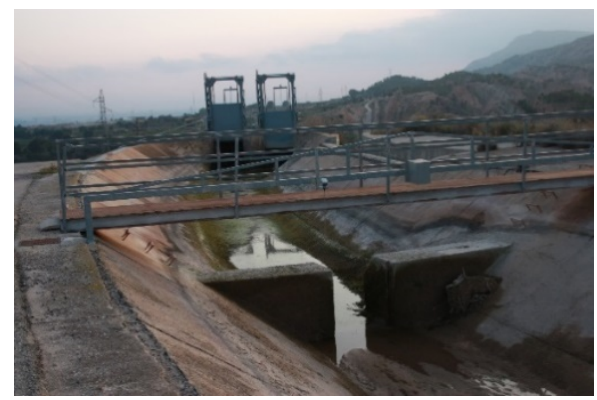

Por otra parte, el agricultor, para acometer reconversiones varietales y cambios de cultivo, que son necesarios para mantener la rentabilidad de la actividad agraria, ha de disponer de un sistema de riego adecuado.

La modernización debía plantearse desde la perspectiva de la instalación de un riego localizado que cubría la superficie regable, abastecido mediante redes de distribución de agua a presión.

El objeto de la obra pasa por optimizar el uso de los recursos actualmente disponibles, incrementar la capacidad de regulación estacional, optimizar las infraestructuras de abastecimiento de agua y energía, con objeto de reducir los costes de impulsión, asegurar el suministro de agua en parcela y automatizar e infor- matizar las infraestructuras hidráulicas, facilitando el control de consumos y la gestión administrativa del agua.

2. Diferenciación de actuaciones y sectores 


\section{Congreso Nacional de Riegos CARTAGENA 2021}

El área regable de la Comunidad está perfectamente definida por el Canal del Trasvase Tajo-Segura Margen Derecha al noroeste, la Rambla de Belén al noreste, el Río Guadalentín al sureste y la Rambla de Algeciras al suroeste;

Analizada el área regable de la Comunidad, con una superficie modernizable total de 2.811,9214 ha, зpara acometer las actuaciones previstas, se dividió en cuatro sectores de riego independientes, cada uno con su toma en el Canal del TTS.

En el presente documento se hablará de sendas actuaciones independientes acometidas en los sectores 2 y 3 .

El Sector 2 , cuenta con una superficie modernizable de 879,5 ha, repartidas en una Zona de Impulsión de 219,3 ha y 371 parcelas reunidas en 43 hidrantes y una Zona de Gravedad de 660,1789 ha y 868 parcelas en 90 hidrantes.
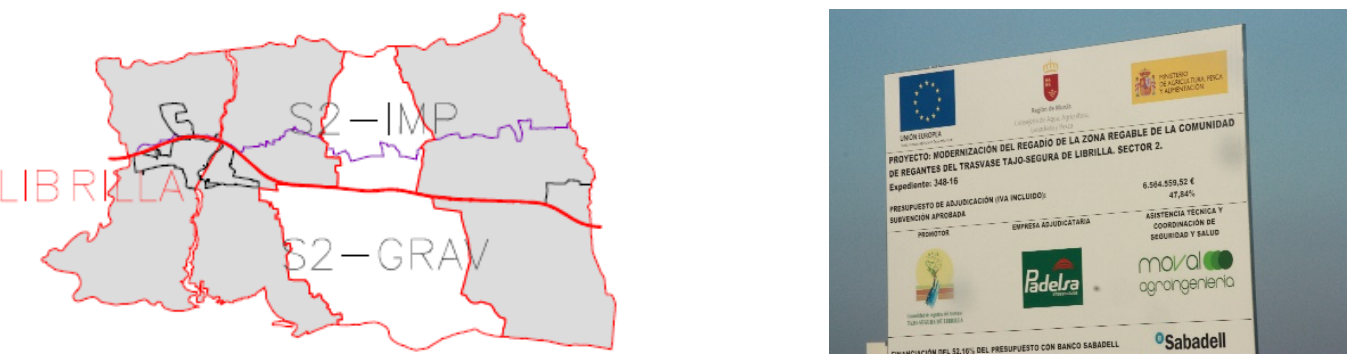

Las obras fueron promovidas a instancia de la Comunidad de Regantes de Aguas del Trasvase TajoSegura de Librilla (Murcia). El proyecto fue redactado por la ingeniería Water Technologies y supervisado por Moval Agroingeniería, quienes a su vez asumieron la Dirección de Obra y la coordinación de seguridad de las obras que fueron ejecutadas por Padelsa. Las obras comenzaron en el año 2018 y finalizaron en Julio del 2020.

El Sector 3 , contaba con una superficie modernizable de 595,88 ha, repartidas en una Zona de Impulsión de 243,9 ha y 574 parcelas reunidas en 67 hidrantes y una Zona de Gravedad de 351,94 ha y 863 parcelas en 97 hidrantes.
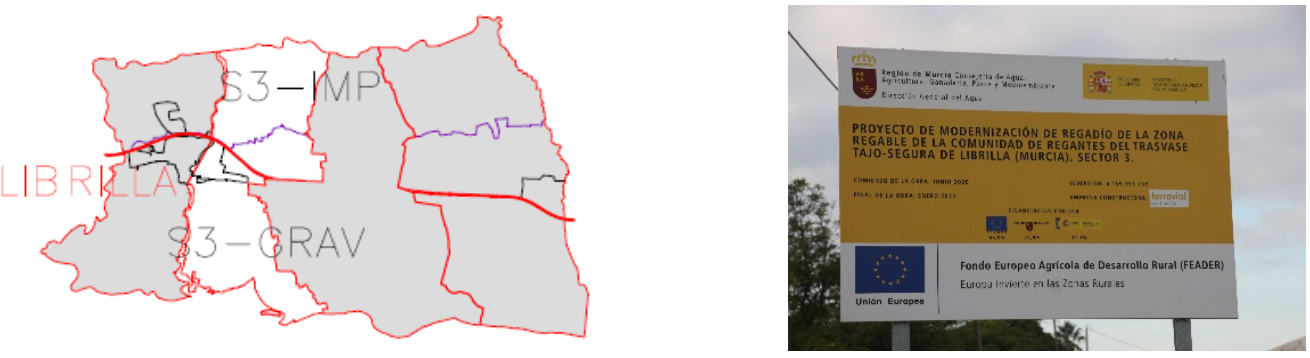

Las obras fueron promovidas a instancia de la Consejería de Agua, Agricultura, Ganadería y Pesca de la Región de Murcia. El proyecto fue redactado por la ingeniería Water Technologies y supervisado por Moval Agroingeniería, quienes a su vez asumieron la Dirección de Obra y la coordinación de seguridad de las obras que fueron ejecutadas por Ferrovial. Las obras comenzaron en junio del año 2020 y tienen prevista su finalización en enero del 2022. 
En ambos casos la supervisión y control se han llevado a cabo por los Servicios Técnicos de la Consejería de la R.M en colaboración con el Servicio Técnico de la Comunidad de Regantes de Librilla.

\section{Diseño de la red y descripción de las obras}

La instalación partirá en cada Sector de un embalse de regulación, que en los Sector 2 y 3 han sido motivo de proyecto aparte y realizado y financiado por la Consejería de agua, Agricultura, Ganadería y Pesca de la Región de Murcia, que se abastecerán desde las correspondientes tomas automatizadas del Canal del TTS.

Cada Sector ésta dividido en dos Zonas, una que necesita aporte de energía (impulsión) y otra que riega por gravedad, y en cada una de ellas, aparte de la automatización, se distinguen dos tipos de actuaciones: la distribución en alta, que comprende todos los elementos desde la toma en el TTS hasta la acometida de los hidrantes colectivos, y la distribución en baja, que considera los necesarios para llegar desde estos últimos hasta todas y cada una de las fincas consideradas.

Para el cálculo de necesidades de agua, se considera representativa del área regable una plantación adulta de limoneros, formada al 60/40 por las variedades Verna y Fino. El diámetro probable del bulbo, dada la textura del terreno, se ha estimado en $1,37 \mathrm{~m}$, lo que supone un área mojada por emisor de 1,47 $\mathrm{m}^{2}$. Con una disposición de 8 emisores de 4 l/h por árbol, considerando un marco de plantación de 5,5x5,5, se obtiene un porcentaje de suelo mojado del $35 \%$. De esta forma se dedujo un módulo de riego de 1,057 1/m²h y así se deduce que es conveniente hacer 3 turnos de riego diarios por cada Zona de cada Sector, y si la JER es de 18 horas, se dispondrá de 6 horas por turno.

La dotación de la que se dispone varía entre los $8.802 .300 \mathrm{~m}^{3} /$ año y $7.310 .740 \mathrm{~m}^{3} / \mathrm{año}$. Esta cifra está conformada por la dotación que tiene la CCRR del Trasvase Tajo-Segura, las escorrentías que se recogen en la PRESA DE LA RAMBLA DE ALGECIRAS, las correspondientes a la concesión de las aguas residuales regeneradas de la EDAR de Librilla. Esta dotación tiene la particularidad de que el reparto es igualitario stanto en calidad como en cantidad, en función de la superficie de cada comunero. Esta singularidad hizo que la automatización prevista fuera a nivel de parcela.
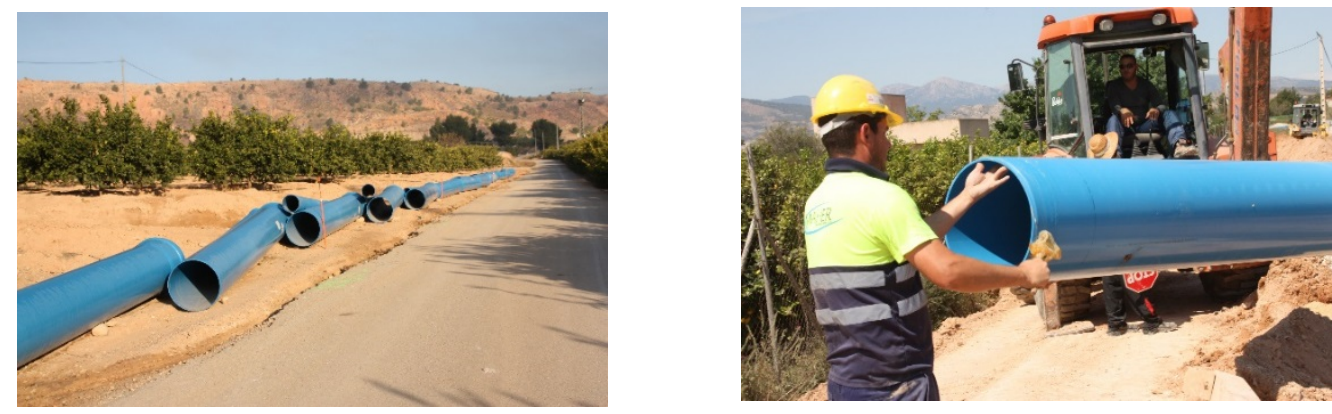

Las parcelas situadas en una franja de aproximadamente $50 \mathrm{~m}$ por debajo de la cota media de cada embalse necesitan del aporte de energía al sistema de riego para su buen funcionamiento. Por lo tanto, los sectores se han dividido en dos Zonas, una que funcionará por impulsión y otra por gravedad. 


\begin{tabular}{ccc}
\hline & SECTOR 2 & SECTOR 3 \\
\hline & \multicolumn{2}{c}{ Superficie (ha) } \\
\cline { 2 - 3 } Impulsión & 219,4 & 243,9 \\
Gravedad & 660,2 & 351,94 \\
Total & 879,6 & 595,84 \\
\hline
\end{tabular}

El funcionamiento de una red en impulsión directa supone el aporte de energía eléctrica para permitir el funcionamiento del equipo de impulsión, que se instalará en los mismos edificios que los cabezales de filtrado. Se utilizará de forma mixta energía de red y energía solar fotovoltaica tanto en la Zona de Impulsión como en la Zona de Gravedad.

A partir del cabezal de filtrado, se ha instalado en cada Zona de riego una red de tuberías principales y terciarias, de diferentes materiales, diámetros y timbrajes, que lleva el agua, filtrada y medida, desde el punto de suministro hasta todas y cada una de las fincas. Las redes principales son ramificadas y rematan en unas casetas o arquetas prefabricadas en donde se albergan los elementos de medición y de automatización en campo de las parcelas (entre 6 y 14 de ellas por caseta o arqueta). Desde las casetas o arquetas hasta las parcelas se han instalado tuberías terciarias independientes para cada una de ellas.

Entre todos los embalses se instalarán tuberías de comunicación para asegurar el trasiego si fuera necesario, que funcionarán por gravedad en el sentido de circulación de agua por el canal y en impulsión aprovechando los grupos motobomba.

\subsection{Cabezales de las Zonas impulsadas}

Bombeos: Con el fin de simplificar trabajos de mantenimiento y de reparación e incluso de reposición de algún grupo motobomba y aunque ello signifique sacrificar algún punto porcentual en cuanto a rendi- miento, se instalaron dos equipos de impulsión exactamente iguales en ambos sectores que cubre distintos turnos de riego, es decir, distintas demandas de caudal y presión. El turno 1 formado por tres grupos motobomba centrífugos horizontales en paralelo en el Sector 2 proporciona 743,719 $\mathrm{m}^{3} / \mathrm{h}$ a una altura manométrica de 69,65 m.c.a mientras que en el Sector 3 el turno 1 proporciona $857,714 \mathrm{~m}^{3} / \mathrm{h}$ a una altura manométrica de 70 m.c.a. Los turnos de bombeo 2 y 3 son cubiertos en ambos sectores por otros tres grupos motobomba centrífugos horizontales de las mismas características, pero con motores de $55 \mathrm{~kW}$ de potencia nominal en paralelo.

Todos los grupos motobomba de cada equipo estarán provistos de un variador de frecuencia hibrido capaz de hacer funcionar los grupos motobomba tanto con la energía de red como a partir de la corriente continua de la energía solar fotovoltaica.

Se instaló una válvula de alivio rápido de presión, que alivia las presiones en el sistema cuando superan un máximo predefinido, abriéndose totalmente y cerrando a continuación con suavidad, con la repetitividad necesaria hasta bajar la presión al nivel de régimen de trabajo, con lo cual constituye un elemento de seguri- dad ante la presencia de sobrepresiones por transitorios rápidos. Así pues, se instaló una válvula de alivio rápido de $200 \mathrm{~mm}$ en derivación, precedida por otra de mariposa para facilitar operaciones de manteni- miento, que verterá por gravedad a un canal principal de riego tradicional próximo al almacén del cabezal. 


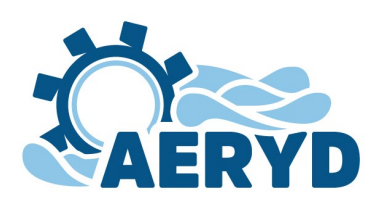

\section{Congreso Nacional de Riegos CARTAGENA 2021}

A la salida de los cabezales se instaló un caudalímetro electromagnético de $400 \mathrm{~mm}$ de diámetro, con el fin de obtener lectura del caudal instantáneo y el volumen suministrado.

Además del bombeo que da servicio a las zonas impulsadas se incluyeron el encargado del aprovechamiento del agua procedente del lavado de filtros en la zona de gravedad en ambos sectores y finalmente, el destinado al aprovechamiento de las aguas residuales regeneradas de la EDAR municipal, que desde su balsa de regulación también se impulsarán hasta el embalse del Sector 3 por el mismo motivo.

En la estación de filtrado de la Zona de Gravedad el agua de la limpieza de filtros se envía a una arqueta de decantación, con un cuerpo de regulación de un volumen de 8,25 $\mathrm{m}^{3}$. Este volumen se vuelve a inyectar a la red de gravedad mediante un grupo motobomba, una vez decantada. Para inyectar este volumen de manera que no se produzcan arranques continuos en el aprovechamiento de estos recursos, se instalará una electro- bomba centrífuga de eje vertical de pequeño caudal, concretamente de 3,5 1/s $\left(12,6 \mathrm{~m}^{3} / \mathrm{h}\right)$, que podrá evacuar el volumen regulado en 0,65 h. La presión necesaria para dicha inyección en la entrada de la instalación de filtrado de la Zona de Gravedad del Sector 2, tendrá que ser superior a 55,5 m.c.a y de 51,5 m en el Sector 3, que es la diferencia de cota entre la lámina de agua superior del embalse de regulación y la del colector de entrada.
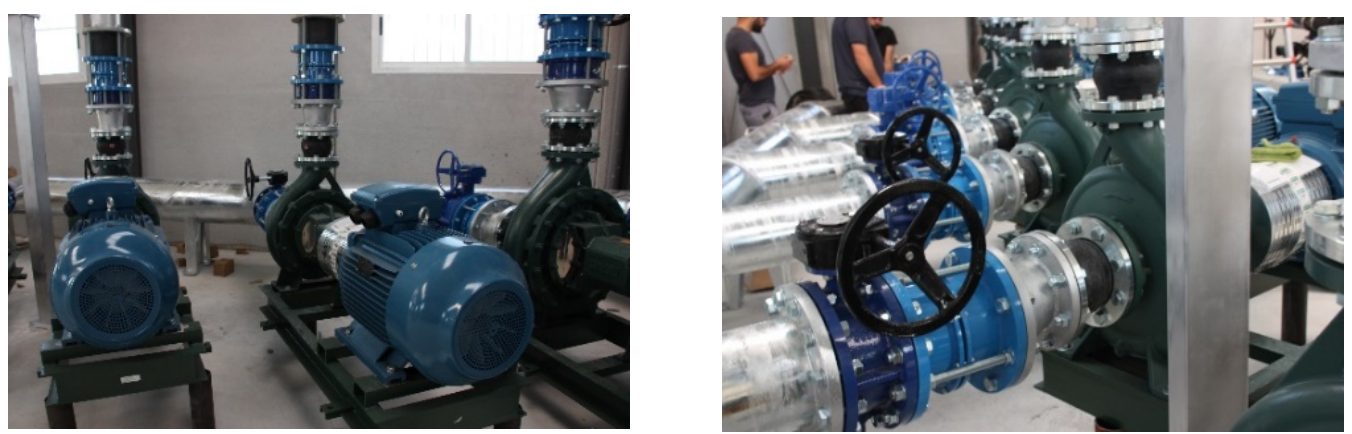

Equipo de filtrado: Formado por 2 baterías de 24 unidades cada una de filtros auto limpiantes de anillas de 3" con un paso de 100 micras, o sistema equivalente que deberá mantener una superficie filtrante de 24,24 m2, todo ello sobre colectores de polipropileno de PN10 y 300 mm de diámetro o de material de igual o mejor fiabilidad.

El lavado se produce secuencialmente de forma automática cuando el presostato diferencial dé lecturas iguales o superiores a $0,5 \mathrm{~kg} / \mathrm{cm}^{2}$.

El sistema de recuperación del agua de limpieza de filtros consiste en la prolongación del colector de salida hasta la coronación del embalse, con 100 m de tubería de PEAD DN110.

Edificio de las estaciones de bombeo: Las bombas y el equipo de filtrado se alojarán en un edificio a pie del embalse con estructura metálica de 25x10 metros en planta para los Sectores 2 y3, cerramiento vertical mediante panel visto prefabricado de hormigón armado y cubierta metálica tipo sándwich. 

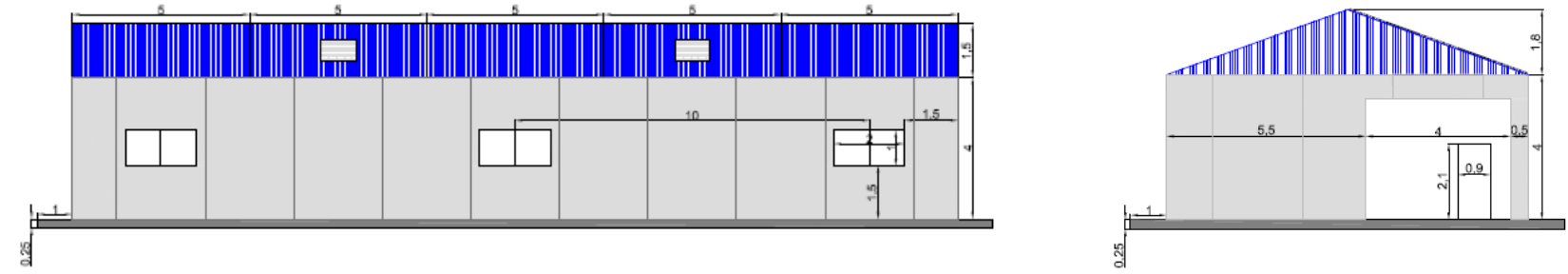

\subsection{Cabezales de las Zonas por gravedad}

Edificio: el equipo de filtrado y demás elementos se alojarán en un edificio con estructura metálica de 20x10 metros en planta y las mismas características constructivas.

Equipo de filtrado: están formados por 2 baterías en el Sector 3 y 5 baterías en el Sector 2 de 32 unidades de filtros de anillas auto limpiantes de 3" y con un paso de filtración de 100 micras o sistema equivalente, todo ello sobre colectores de polipropileno de PN10 y $350 \mathrm{~mm}$ de diámetro.

Se construyó una arqueta semienterrada de recogida del agua de limpieza de filtros de 8,25 $\mathrm{m}^{3}$ de capacidad de regulación y se instalará un grupo motobomba de impulsión multicelular de eje vertical, capaz de inyectar un caudal nominal de $12,6 \mathrm{~m}^{3} / \mathrm{h}$ a una presión de 60 m.c.a en el colector de entrada del equipo de filtrado, con una potencia nominal de $4 \mathrm{~kW}$.

Se instaló también una válvula de alivio de $250 \mathrm{~mm}$ de diámetro que vierte a la arqueta de recuperación del agua de lavado de filtros.

A la salida del cabezal se instaló un caudalímetro electromagnético de $600 \mathrm{~mm}$ de diámetro, con el fin de obtener lectura del caudal instantáneo y el volumen suministrado.

\subsection{Suministro de Energía}

El suministro de energía de red a los cabezales de impulsión se hizo con una línea aérea de media tensión de 312,2 metros para el Sector 2 y de 407,6 m para el Sector 3, línea subterránea de media tensión de entrada al centro de transformación del tipo intemperie compacto (CTIC), de $250 \mathrm{KVA}$ de potencia, e instalación en baja tensión.

El suministro de energía solar fotovoltaica en la zona de impulsión se hará a partir de sendas plantas aisladas de suelo de 594 paneles para el Sector 2 y 378 para el Sector 3 de $300 \mathrm{Wp}$, de una potencia de 178,2 kWp, que será capaz de proporcionar anualmente 257.570,2 kWh/año en el S2 y 145.880,8 kWh/año en el S3. El suministro de los cabezales de la Zona de Gravedad de menor demanda energética se hizo de igual manera para ambos sectores, mediante una planta solar fotovoltaica de techo, formada por 40 paneles de 300 Wp y una

potencia de $12 \mathrm{kWp}$, capaz de proporcionar la energía eléctrica necesaria para todos los puntos de consumo del edificio a lo largo del día, y conectada a la red para garantizar el suministro cuando no haya producción solar. La energía sobrante fotovoltaica se inyectará a la red, compensando el consumo.

Desde el centro de transformación y desde cada cuadro central de las plantas generadoras de energía fotovoltaica, bien sean de suelo o de techo, parten las líneas de acometida en baja tensión hasta los Cuadros Generales de Protección de las estaciones de bombeo de los cabezales de la Zona de Impulsión y al cabezal de filtrado de las Zonas de Gravedad. 


\subsection{Redes principales de distribución de agua}

Tuberías: Para llevar el agua desde los cabezales hasta los hidrantes colectivos de cada una de las dos Zonas, se proyectan redes de distribución ramificadas.

La presión necesaria en cada hidrante serán las alturas piezométricas calculadas para cada uno, más la pérdida de carga considerada en su interior (5 m.c.a).

La dotación de riego adoptada de 1,057 1/ $\mathrm{m}^{2}$.h es el resultado del diseño agronómico estudiado.

En el proyecto se incluyó un estudio comparativo entre los diferentes materiales que se pueden utilizar para las conducciones, teniendo en cuenta su precio instalado y el zanjeo, anclajes y capacidad hidráulica. De él se deduce que, para diámetros de $140 \mathrm{~mm}$ o superiores, el material indicado es el PVC-O serie 500, y entre 125 mm y 90 mm, tomado como diámetro inferior admisible, el material idóneo es el PEAD PE100.

Las mediciones de tubería por materiales instaladas son:

- SECTOR 2: $25.283 \mathrm{~m}$ de longitud en PVC-O serie 500 y $4.673 \mathrm{~m}$ en PEAD (PE-100)

- SECTOR 3 :22.301 m de longitud en PVC-O serie 500 y 4.479 m en PEAD (PE-100)

\begin{tabular}{|c|c|c|c|c|c|c|c|c|c|}
\hline \multicolumn{9}{|c|}{ TUBERÍAS RED PRINCIPAL PVC-O SECTOR 2} & \multirow{3}{*}{$\begin{array}{c}\text { TOTAL } \\
\text { MEDICIÓN } \\
\text { PVC-O (m) }\end{array}$} \\
\hline \multirow{2}{*}{ RED } & \multirow{2}{*}{ TIMBRAJE } & \multicolumn{6}{|c|}{$\varnothing$} & & \\
\hline & & 140 & 160 & 200 & 225 & 250 & 315 & & \\
\hline \multirow{2}{*}{ IMPULSIÓN } & PN12,5 & 209 & 102 & 958 & & 837 & 36 & & \multirow{11}{*}{25.283} \\
\hline & PN16 & 413 & 448 & 365 & 227 & 451 & 153 & & \\
\hline \multirow{2}{*}{ GRAVEDAD } & PN12,5 & 1.498 & 1.048 & 276 & 1.089 & 691 & 3.154 & & \\
\hline & PN16 & 143 & 617 & 1.081 & 213 & 419 & & & \\
\hline \multicolumn{2}{|c|}{ TOTAL (m) } & 2.263 & 2.215 & 2.680 & 1.529 & 2.398 & 3.343 & & \\
\hline \multirow{2}{*}{ RED } & \multirow{2}{*}{ TIMBRAJE } & \multicolumn{6}{|c|}{$\emptyset$} & \multirow[b]{2}{*}{800} & \\
\hline & & 355 & 400 & 450 & 500 & 630 & 710 & & \\
\hline \multirow{2}{*}{ IMPULSIÓN } & PN12,5 & 657 & 576 & & & & & & \\
\hline & PN16 & 621 & & & & & & & \\
\hline \multirow{2}{*}{ GRAVEDAD } & PN12,5 & 1.003 & 913 & 654 & 1.320 & 2.338 & 1.378 & 441 & \\
\hline & PN16 & 710 & 244 & & & & & & \\
\hline \multicolumn{2}{|c|}{ TOTAL (m) } & 2.991 & 1.733 & 654 & 1.320 & 2.338 & 1.378 & 441 & \\
\hline
\end{tabular}

\begin{tabular}{|c|c|c|c|c|c|c|c|c|}
\hline \multicolumn{8}{|c|}{ TUBERÍAS RED PRINCIPAL PVC-O SECTOR 3} & \multirow{3}{*}{$\begin{array}{c}\text { TOTAL } \\
\text { MEDICIÓN } \\
\text { PVC-O (m) }\end{array}$} \\
\hline \multirow{2}{*}{ RED } & \multirow{2}{*}{ TIMBRAJE } & \multicolumn{6}{|c|}{$\varnothing$} & \\
\hline & & 140 & 160 & 200 & 225 & 250 & 315 & \\
\hline \multirow{2}{*}{ IMPULSIÓN } & PN12,5 & 606 & 147 & 236 & 845 & 275 & 875 & \multirow{14}{*}{22.301} \\
\hline & PN16 & 721 & 358 & 349 & 1.195 & 183 & 143 & \\
\hline \multirow{2}{*}{ EDAR } & PN12,5 & & & & & & 1.600 & \\
\hline & PN16 & & & & & & 2.404 & \\
\hline \multirow{2}{*}{ GRAVEDAD } & PN12,5 & 1.427 & 705 & 584 & 2.077 & 216 & & \\
\hline & PN16 & 149 & & & & & & \\
\hline \multicolumn{2}{|c|}{ TOTAL (m) } & 2.903 & 1.209 & 1.169 & 4.116 & 673 & 5.021 & \\
\hline \multirow{2}{*}{ RED } & \multirow{2}{*}{ TIMBRAJE } & \multicolumn{6}{|c|}{$\emptyset$} & \\
\hline & & 355 & 400 & 450 & 500 & 630 & 800 & \\
\hline \multirow{2}{*}{ IMPULSIÓN } & \begin{tabular}{|l|} 
PN12,5 \\
\end{tabular} & 146 & 98 & & & & & \\
\hline & PN16 & & & & & & & \\
\hline \multirow{2}{*}{ GRAVEDAD } & \begin{tabular}{|l|} 
PN12,5 \\
\end{tabular} & 1.795 & 667 & 862 & 420 & \begin{tabular}{|l|}
3.221 \\
\end{tabular} & & \\
\hline & PN16 & & & & & & & \\
\hline \multicolumn{2}{|c|}{ TOTAL (m) } & 1.942 & 764 & 862 & 420 & 3.221 & 0 & \\
\hline
\end{tabular}

\begin{tabular}{|c|c|c|c|c|c|c|c|}
\hline \multicolumn{7}{|c|}{ TUBERÍAS RED PRINCIPAL PEAD } & \multirow{3}{*}{$\begin{array}{c}\text { TOTAL } \\
\text { MEDICIÓN } \\
\text { PEAD (m) }\end{array}$} \\
\hline \multirow{2}{*}{ RED } & \multirow{2}{*}{ TIMBRAJE } & \multicolumn{5}{|c|}{$\varnothing$} & \\
\hline & & 90 & 110 & 125 & & & \\
\hline \multirow{2}{*}{ IMPULSIÓN } & PN10 & & 286 & 668 & & & \multirow{5}{*}{4.673} \\
\hline & PN16 & 112 & 519 & 547 & & & \\
\hline \multirow{2}{*}{ GRAVEDAD } & PN10 & 41 & 981 & 1.208 & & & \\
\hline & PN16 & 311 & & & & & \\
\hline \multicolumn{2}{|c|}{ TOTAL (m) } & 464 & 1.786 & \multicolumn{3}{|l|}{2.423} & \\
\hline \multicolumn{7}{|c|}{ TUBERÍAS RED PRINCIPAL PEAD } & \multirow{10}{*}{4.479} \\
\hline \multirow{2}{*}{ RED } & \multirow{2}{*}{ TIMBRAJE } & \multicolumn{5}{|c|}{$\varnothing$} & \\
\hline & & 90 & 110 & 125 & 315 & 500 & \\
\hline \multirow{2}{*}{ IMPULSIÓN } & PN10 & 206 & 96 & 179 & & & \\
\hline & PN16 & 401 & 348 & 375 & & & \\
\hline \multirow{2}{*}{ EDAR } & PN10 & & & & & 91 & \\
\hline & PN16 & & & & 91 & & \\
\hline \multirow{2}{*}{ GRAVEDAD } & PN10 & 468 & 1.269 & 956 & & & \\
\hline & \begin{tabular}{|l|} 
PN16 \\
\end{tabular} & & & & & & \\
\hline \multicolumn{2}{|c|}{ TOTAL (m) } & 1.075 & 1.713 & 1.510 & 91 & 91 & \\
\hline
\end{tabular}



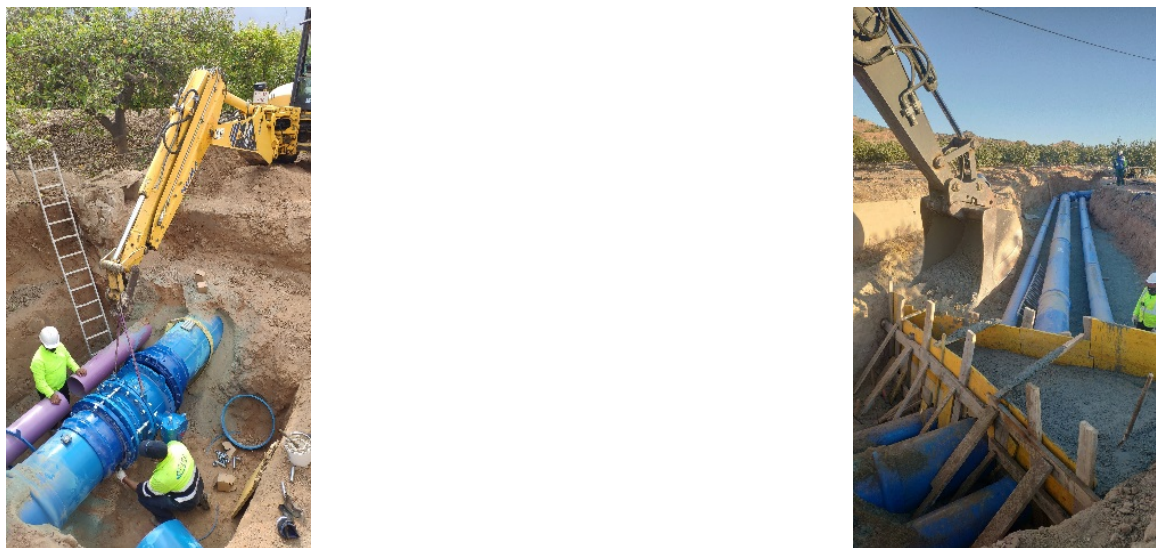

Hidrantes colectivos e individuales: Se han instalado 133 hidrantes colectivos en el sector 2 y 158 en el Sector 3. Se cuenta además con un total de 574 hidrantes individuales en la Zona de Impulsión y de 863 en la

de Gravedad del Sector 2 y 371 en la Zona de Impulsión y 868 en la de Gravedad en el Sector 3.

Los Individuales están formados por un contador con emisor de pulsos y una electroválvula hidráulica de membrana con un piloto reductor de presión, que sirve para controlar el flujo de agua de riego que abastece a una finca determinada, en el ámbito del hidrante colectivo al que pertenece.

Los hidrantes colectivos están formados por válvulas de descarga, válvulas de mariposa, filtros cazapiedras, colectores multitoma, ventosas y manómetros o presostatos. Además de los accesorios y piezas especiales necesarios para su instalación o conexión.

Tuberias Terciarias: son las encarg

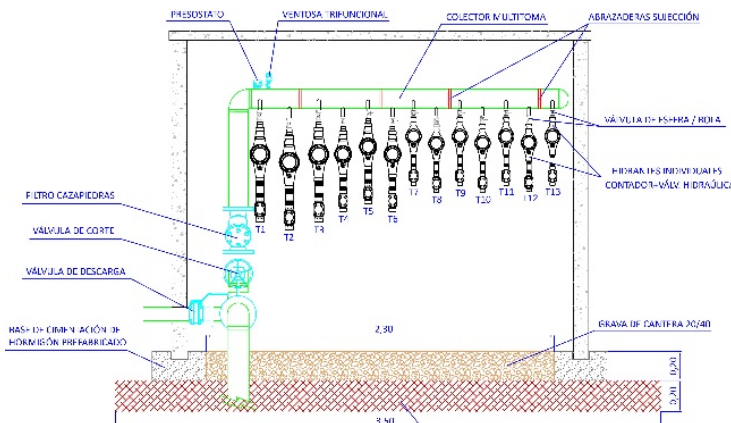

finca. Son de PEAD de diámetros comprendidos entre $32 \mathrm{~mm}$ y $250 \mathrm{~mm}$ con una longitud aproximada

cela o

km en el Sector 2 y 193 km en el Sector 3 de tal manera que la presión mínima disponible en el punto

más alto de cada parcela será de 25 m.c.a.

\subsection{Tubería de impulsión y grupo de bombeo edar del Sector 3}

En el embalse de la EDAR se recogen las aguas de la misma, con un caudal ficticio continuo de 11,01 1/s, pero la posibilidad de aumento de concesión y la necesidad de tener que vaciar la balsa en aproximada- mente 2 días, hace que se considere un caudal de 90 l/s. Para su aprovechamiento es necesaria una impulsión que las lleve al embalse de regulación del Sector 3, el más asequible, para desde allí distribuirlas a los demás embalses, si conviene para unificar su calidad. 
En este caso se dispone en la parcela de energía de red suficiente para suministrar al equipo de impulsión formado por tres grupos motobomba centrífugos horizontales en paralelo, capaces de bombear cada uno un caudal de 31,1 1/s a una altura de 127 m.c.a con motor eléctrico de $55 \mathrm{~kW}$ de potencia nominal y variadores de frecuencia. La tubería de impulsión, de 4.340 m de longitud total es de PVC-O PN 16/12,5, de 315 mm de diámetro.
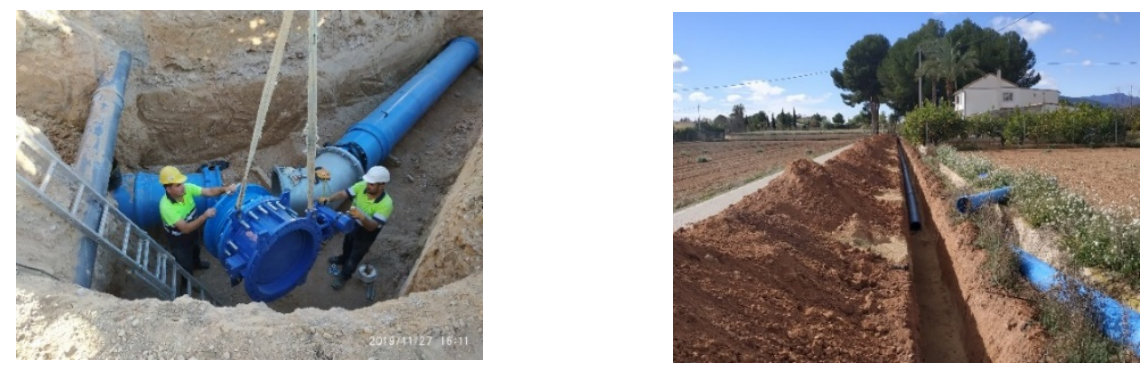

\subsection{Tubería de trasiego entre Sectores de riego:}

De una longitud de 1.924,50 m, formada por tubos de PVC-O de $400 \mathrm{~mm}$ de diámetro PN12,5. Para poder llevar agua desde el embalse de regulación del Sector 1 hasta él del Sector 2 por gravedad y desde éste último hasta el primero, bien por gravedad si las láminas de agua de ambos lo permiten o mediante los equipos de impulsión del Sector 2 si no es así, se instalará una tubería de PVC-O de $400 \mathrm{~mm}$ de diámetro en PN12,5 entre las arquetas de salida de ambos embalses, que tendrá una longitud total de 1.924,5 m, cuya traza puede coincidir con alguna línea de la red de distribución de las Zonas de Impulsión de cualquiera de los dos Sectores, o con la tubería de transporte a EF-2. De momento solo se ha instalado la parte que corresponde al ámbito del Sector 2, ya que el tramo desde el Sector 2 al Sector 3, e incluso el tramo desde es Sector 4 al Sector 3 ya estaban realizados.

\subsection{Automatización}

Se han instalado unidades remotas para control de hidrantes y valvulería, que comunicarán con unidades concentradoras (una en la zona de impulsión y otra en la de gravedad) vía radio.
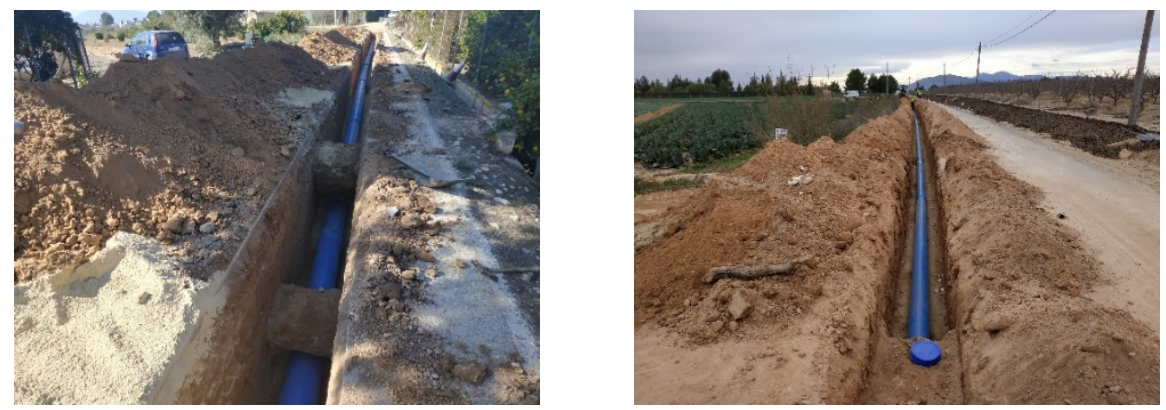

Desde un Centro de Control ubicado en la oficina central de la Comunidad, sita en C/ Murcia, no 32, Librilla (Murcia), se comandan y gestionan todos los elementos a automatizar, acción que es redundante, (wifi y gprs) desde las concentradoras instaladas en las edificaciones que alojan las estaciones de filtrado, donde se dispone de energía procedente de la red eléctrica o solar fotovoltaica. 


\section{Congreso Nacional de Riegos CARTAGENA 2021}

\section{Conclusiones}

Creemos necesario recordar que el ahorro y eficiencia energética de una Modernización de Regadíos comienzan en la fase de diseño de la actuación, tanto del esquema hidráulico como en la elección de los materiales de la red, y esta actuación es un buen ejemplo de ello.

De no ser así, se estará condenado únicamente a la adopción de medidas sólo paliativas que tendrán una menor repercusión en la factura eléctrica, que, desde luego, no desdeñamos, pero consideramos insuficientes.

Medidas tales como la ejecución de balsas de acumulación para aprovechar el caudal que se pueda bombear en horas con menor coste energético el aprovechamiento, donde sea posible, de los desniveles naturales que puedan reducir los costes de impulsión, el uso de energías renovables y por fin, el uso de materiales eficientes que permitan, debido a sus características intrínsecas, el transporte del agua de riego con la menor pérdida de carga posible, han de primar a la hora de la valoración de la alternativa a acometer.

Es pues el marco actual, el más apropiado para el uso del PVC-O y sus accesorios en las redes hidráuli- cas de las nuevas modernizaciones. Un material, que debido a sus propiedades genera una menor pérdida de carga y consecuentemente de energía respecto otros materiales y permite un ahorro considerable de la potencia necesaria a instalar y posteriormente contratar y del propio consumo de energía en estas actuaciones.

\section{Referencias}

1. Water Technologies. Proyectos constructivos de los Sectores 2 Y 3. Proyecto de Modernización del Regadío de la Zona Regable de la Comunidad de Regantes del Trasvase Tajo - Segura de Librilla. sector 3 y Proyecto de Modernización del Regadío de la Zona Regable de la Comunidad de Regantes del Trasvase Tajo-Segura de Librilla. sector 2. 